\title{
Métodos y técnicas para la construcción de símbolos táctiles hacia una Cartografía Inclusiva
}

\author{
Enrique Pérez de Prada* \\ Waldirene Ribeiro do Carmo** \\ Carla R.G. Sena***
}

Recibido 25 de mayo de 2019; aceptado 24 de junio de 2019

\begin{abstract}
This article presents an overview of the experiences over several years of the authors involving the production of tactile graphic representations, with emphasis on creating the symbols (linear, area and point) used to portray geocartographic information. The symbols that are already standardized will be presented, as will the methodologies used for defining and setting them up. Making use of the symbology of tactile maps for tourism as an example, the features necessary for signs to be understood by the users, mainly those who have visual deficiencies, will be discussed.

A tactile cartographic product makes it possible for visually disabled persons to have access to geographic information and knowledge by means of the tactile symbols and the Braille text that are included in each of the cartographic products. This type of tactile cartography enables one to portray information in a way that is suitable and understandable for the blind user, which involves using carefully designed tactile symbols. This lets them find, get to know and set up a mental image of the geographic area portrayed.

It is important to point out that an adequate teaching of tactile reading and of the Braille language, along with the prior knowledge held by the blind person about the geographic variable portrayed, will make it easier for that person to extract information from a tactile map, from a graphic image or from images in relief.

Key words: tactile symbols, tactile maps, blind, low vision, cartography.

* Centro de Cartografía Táctil, Facultad de Humanidades y Tecnologías de la Comunicación Social, Universidad Tecnológica Metropolitana, Chile, correo electrónico: eperez@utem.cl

** Departamento de Geografía, Universidad de Sao Paulo, Brasil, correo electrónico: wal.carmo@gmail.com

***Universidad Estadual Paulista, Ourinhons, Sao Paulo, Brasil, correo electrónico: cacrisusp@gmail.com
\end{abstract}




\section{Resumen}

Este artículo presenta una reflexión sobre la experiencia de varios años de los autores con relación a la producción de representaciones gráficas táctiles, con énfasis en la generación de los símbolos (lineales, areales y puntuales) empleados para representar informaciones geocartográficas. Se presentarán los símbolos que ya están estandarizados, así como las metodologías utilizadas para su definición y construcción. Aprovechando como ejemplo la simbología de algunos mapas táctiles turísticos serán discutidas las características necesarias para que los signos sean comprendidos por los usuarios, principalmente los que tienen deficiencia visual.

Un producto cartográfico táctil, permite al discapacitado visual acceder a la información y el conocimiento geográfico por medio de los símbolos táctiles y la escritura Braille que acompaña a cada uno de los productos cartográficos. Este tipo de cartografía permite representar la información de una forma adecuada y entendible para el usuario ciego, lo cual implica utilizar símbolos táctiles cuidadosamente diseñados, lo que permitirá reconocer y generar una imagen mental del área geográfica representada.

Es importante señalar, que la adecuada instrucción en la lectura táctil, en el lenguaje Braille y el conocimiento previo de la variable geográfica representada que tenga la persona ciega, le facilitará extraer información de un mapa táctil, de un gráfico o de imágenes en relieve.

Palabras claves: símbolos táctiles, mapas táctiles, ciegos, baja visión, cartografía.

\section{Resumo}

Este artigo apresenta uma reflexão sobre a experiência de vários anos dos autores com relação à produção de representações gráficas táteis, com destaque na criação de símbolos (lineares, areais e pontuais) empregados para representar informações geocartográficas. Serão apresentados os símbolos que já estão padronizados, assim como as metodologias utilizadas para sua definição e construção. Aproveitando como exemplo a simbologia de alguns mapas táteis turísticos serão discutidas as características necessárias para que os signos sejam compreendidos pelos usuários, principalmente os que têm deficiência visual.

Um produto cartográfico tátil, permite que as pessoas com deficiência visual acessem informações e conhecimento geográfico por meio dos símbolos táteis e da escrita em braile que acompanha cada um dos produtos cartográficos. Este tipo de cartografia tátil permite representar a informação de uma forma adequada e compreensível para o usuário com deficiência visual, o que implica a utilização de símbolos táteis cuidadosamente pensados, que permitirão reconhecer e gerar uma imagem mental da área geográfica representada. 
É importante ressaltar que a experiência em leitura tátil e na linguagem em braile, assim como o conhecimento prévio que tenha a pessoa com deficiência visual sobre a variável geográfica representada, facilitará a obtenção de informações de um mapa tátil, de um gráfico ou de imagens em relevo.

Palavras chave: símbolos táteis, mapas táteis, pessoas com deficiência visual, cartografia.

\section{Introducción}

En las últimas décadas, los cartógrafos han estudiado las bases teóricas de la Cartografía y han propuesto diversas definiciones, demostrando que no hay consenso en cuanto a los conceptos, producción y uso de los mapas, lo que en parte compromete los avances de la disciplina y sus relaciones con otros campos como los Sistemas Geográficos de la Información (SIG). La definición más reciente divulgada en el Plan Estratégico (2003-2011) de la Asociación Cartográfica Internacional (ICA) de resume Cartografía como "una disciplina que involucra el arte, la ciencia y la tecnología de producir y usar mapas". (ICA, 2011). En la actualidad, existe una Agenda de Investigación para la Cartografía y las Ciencias de la Información Geográfica (GIScience) (Virrantaus et al., 2009) y un nuevo Plan Estratégico 20112019 que incluye repensar las bases teóricas y prácticas, los conceptos y el alcance de la disciplina, señalando que el objetivo de la ICA es garantizar que la Cartografía y la GIScience, sean empleadas para el máximo efecto y pleno potencial en el beneficio de la sociedad y de la ciencia a través de la promoción y representación de las disciplinas y profesiones de la Cartografía y de la GIScience internacionalmente (ICA, 2011). En conferencia de ICA "MAPEANDO TODO PARA TODOS", celebrada en la ciudad de Tokio (julio, 2019), se abordaron los temas mencionados en el presente texto (</https://icaci.org/icc2019/>).

Basaraner (2016) presenta una excelente visión general sobre la evolución de los conceptos, técnicas y paradigmas de la cartografía y una lista extensa de cartógrafos que contribuyeron a la disciplina. El autor discute estos diversos conceptos y paradigmas que han surgido en el campo de la Cartografía y destaca algunos de los más importantes en el área: comunicación cartográfica, semiología gráfica, cartografía analítica, geovisualización, Cibercartografía, cartografía web/móvil/ omnipresente y neocartografía, entre otros. Destaca también los tres grandes paradigmas de esa ciencia, 1) la producción, 2) los analíticos y 3) los de la comunicación (cognitivos). Basaraner (op. cit.) analiza en profundidad la relación entre Cartografía y el tratamiento, gestión y uso de la información espacial, proponiendo un marco teórico moderno y amplio para la cartografía (Figura 1). 


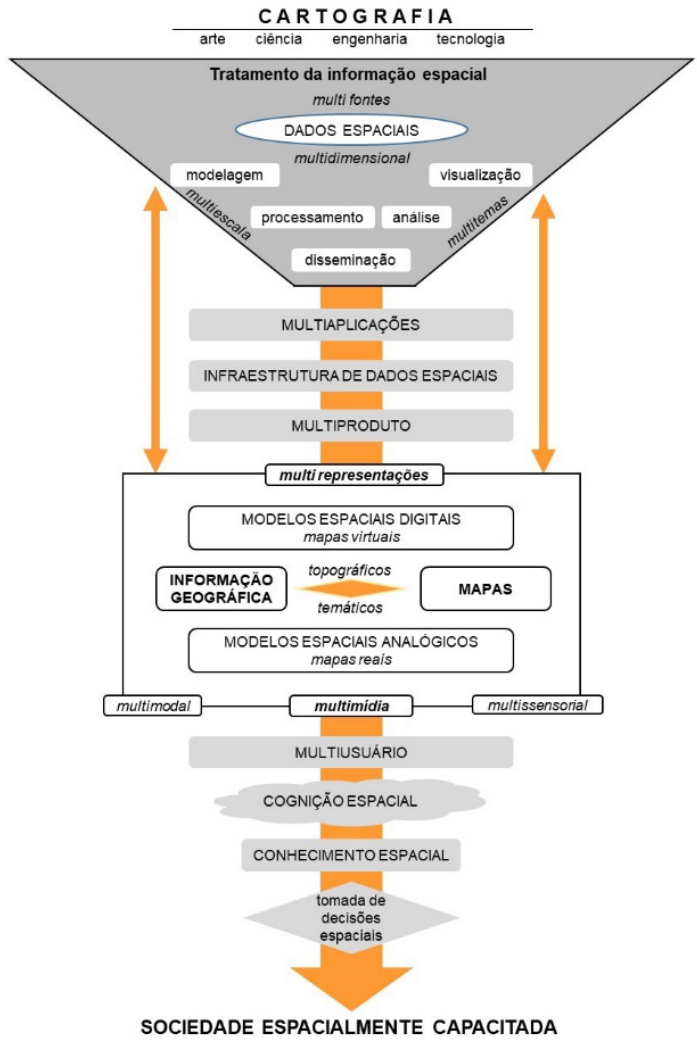

Figura 1. Un marco teórico moderno y completo para la Cartografía. Fuente. Adaptado de Basaraner (2016: 85).

En la Figura 1, el autor presenta el campo de la cartografía, en un intento de identificar y desarrollar un cuadro teórico actual y amplio para la cartografía, revisitando conceptos, técnicas y paradigmas e investigando sus relaciones con el tratamiento y uso de las informaciones espaciales. Se nota la repetición de la palabra "multi" en las diversas etapas, lo que va al encuentro de las ideas de los autores y de las propuestas de una cartografía inclusiva, multisensorial, multimodal e interactiva, también en el sentido de alcanzar una sociedad espacialmente capacitada. Para Basaraner (op. cit.), la cartografía es una disciplina espacial esencial para la Tierra y para la sociedad que precisa y merece ser identificada en un contexto más amplio, con todas sus aplicaciones.

La Figura 2 presenta un marco teórico para la Cartografía Inclusiva (Almeida, 2014). Esta nueva estructura difiere de las propuestas anteriores (Vasconcellos, 1996; Almeida, 2011), en la medida en que incluye perspectivas y dimensiones dirigidas a una cartografía para todos, incorporando las tecnologías de la información y 
comunicación actuales. Por ejemplo, cuatro perspectivas se destacan (cultural, social, inclusiva y política) junto con cuatro dimensiones, que son: diseño y arte, comunicación, tecnología y ubicuidad (omnipresencia). Las últimas cuatro se consideran vitales para la cartografía en los días de hoy. Los mismos elementos de la comunicación cartográfica continúan en pauta: por quién, qué, por qué, cómo, a través de qué signos, medios y dispositivos, para quién y con qué resultados.

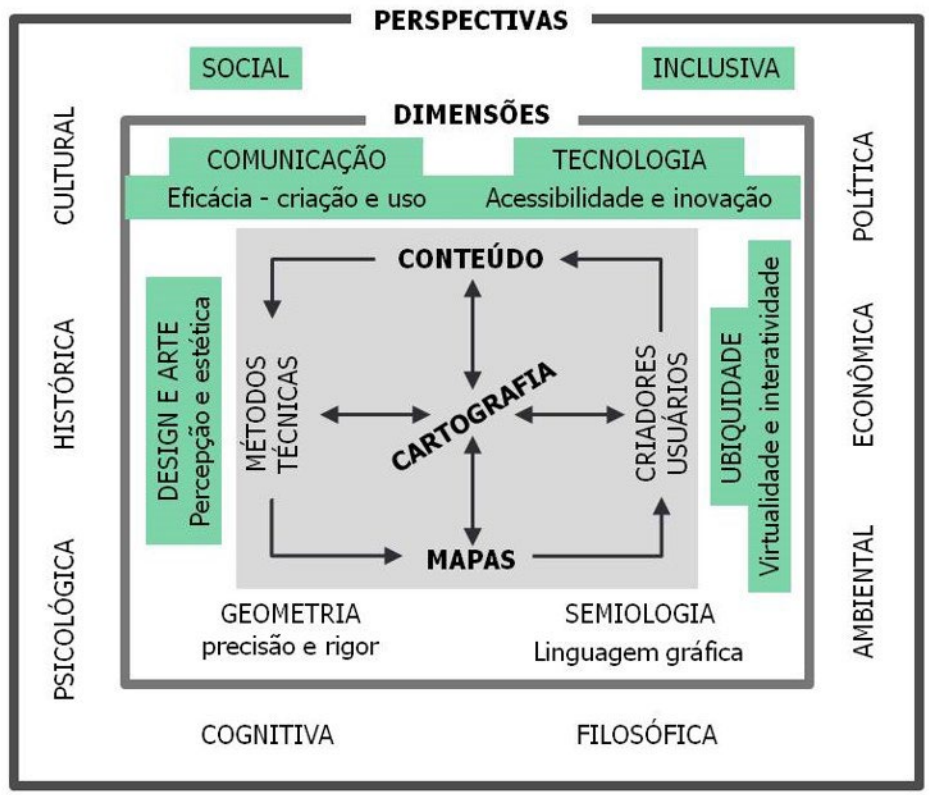

Figura 2. Marco teórico para una Cartografía Interactiva e Inclusiva.

Fuente: Adaptada de Almeida (2014: 110).

Vasconcellos (1993) en su tesis de doctorado presentó una propuesta de transformar las variables visuales formuladas por Jacques Bertin (1977) en variables táctiles. Las variables visuales son signos y signos abstractos que construyen la representación gráfica-cartográfica. La semiología gráfica permite expresar de forma lógica y estética cualquier fenómeno de la superficie terrestre, pero como sus componentes son básicamente visuales fue necesario transferir estas características a la percepción táctil.

La autora desarrolló también un programa de introducción al uso de los mapas. Este programa incluye la presentación de nociones geográficas básicas, tales como, escala, distancia, ubicación, dirección y orientación, desarrollados por medio de una serie de actividades que tuvieron como objetivo no sólo ayudar a los estudiantes a 
comprender el lenguaje simbólico de los mapas, sino también despertar el interés y motivarlos, con el uso de juegos e historias. La autora cree que es fundamental "seducir" a los alumnos y que un camino es desarrollar la percepción, usando todos los sentidos para aprender.

Para la elaboración de representaciones táctiles de calidad que realmente puedan comunicar la información deseada, se utilizaron varias técnicas de construcción de mapas en relieve donde se utilizaron diferentes tipos de símbolos y mapas. La investigación llevada a cabo en proyectos apoyados por el Instituto Panamericano de Geografía e Historia (IPGH), desde el año 1994, permitió construir y evaluar numerosos mapas táctiles, y así seleccionar los materiales y especialmente los símbolos más eficientes para cada información que se pretendía representar.

Este artículo pretende exponer algunos símbolos táctiles estandarizados a partir de las evaluaciones llevadas a cabo en Argentina, Brasil, Chile y Perú, con estudiantes tanto ciegos como de baja visión y las técnicas más utilizadas para su producción.

\section{Técnicas de construcción de representaciones táctiles}

A continuación las técnicas de construcción de representaciones táctiles desarrolladas y ampliamente evaluadas hasta ese momento. Los recursos didácticos para la enseñanza de Geografía fueron hechos a partir de los principios de la cartografía táctil y originalmente fueron pensados para estudiantes con deficiencia visual, pero con el uso en las salas regulares, se notó un gran interés para utilizar este material por todos los estudiantes.

Los mapas y gráficos táctiles pueden ser construidos utilizando técnicas artesanales simples con pegamento (Figura 3), que presenta la ventaja de poder agregar las más variadas texturas para la representación de la información, sea en la implantación puntual, lineal o zonal. Por ejemplo, puntos representados por perlas y botones, líneas, soutache y cordones; áreas de rejillas de tejido, lijas, papel de cartón, arena o cualquier otra textura. Si la representación es elaborada para ser utilizada como producto final por los estudiantes se pueden incorporar otros materiales tales como espuma de poliestireno, goma EVA (caucho sintético generalmente comercializado en láminas de diversos espesores), plásticos y tinta en relieve para tejido. Esta gran disponibilidad de texturas permite la representación de más variables táctiles en cada mapa, pero la técnica del collage obliga a una generalización mayor de la información a ser representada ya que algunos materiales son difíciles para el corte en dibujos con muchas curvas o detalles pequeños.

Esta técnica no requiere gran habilidad en diseño, pero demanda un tiempo mayor para producción del mapa debido a la especificidad de cada material trabajado. Además, el producto final se deteriora más rápidamente cuando se expone al uso 


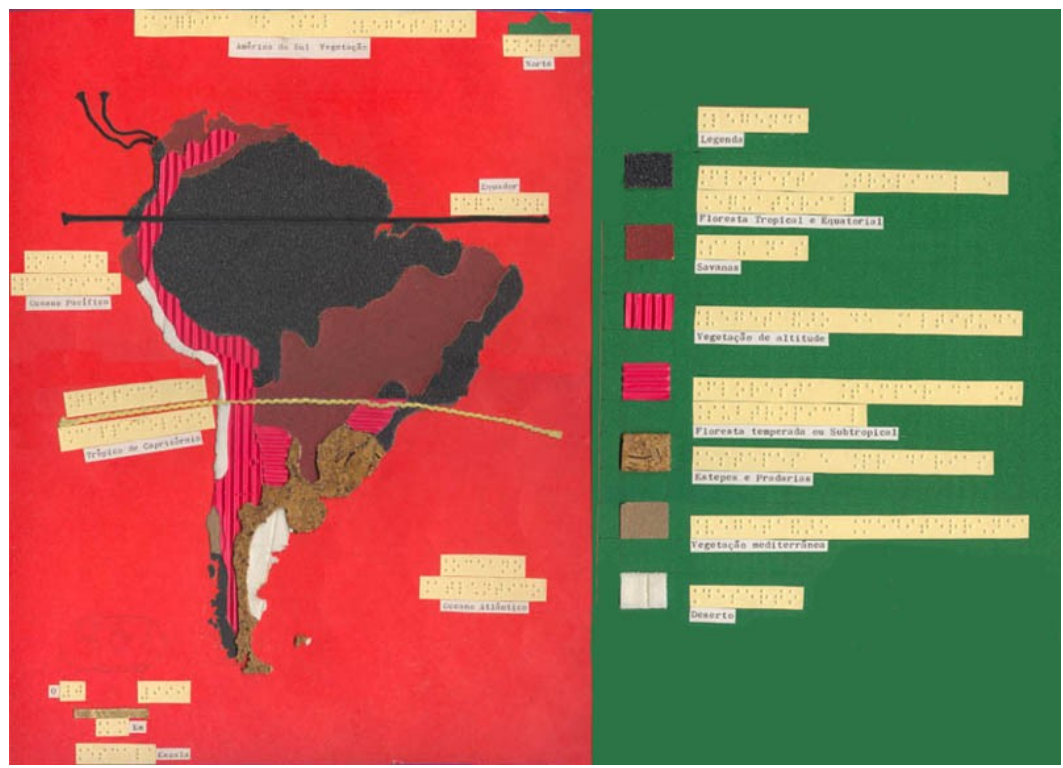

Figura 3. América del Sur. Vegetación. Mapa en collage.

Fuente: Registro de Mapas Táctiles del LEMADI, USP.

directo. Por este motivo, estas representaciones táctiles son generalmente producidas como matrices, así el tiempo gastado en su confección recompensa por el gran número de copias que es posible sacar. Sin embargo, es necesario considerar que generalmente los mapas artesanales hechos con pegamento son termoformados en plástico en una máquina de moldeo al vacío (en esa máquina, la lámina de plástico es calentada, posteriormente es succionada sobre la matriz y adquiere la forma que se diseñó) por eso es necesario utilizar materiales resistentes al calor.

Otra técnica considerada artesanal consiste en el uso de láminas de aluminio (Figura 4a, b y c) de espesor 0.10 a $0.14 \mathrm{~mm}$ que, apoyadas en una superficie blanda, son esculpidas con espátulas, carretes o incluso la punta de un bolígrafo. Esta técnica es ideal para la realización de dibujos simples, representaciones lineales y con poca variación de texturas. Su elaboración es rápida, pero depende un poco de la práctica con el uso de las herramientas presentándose como una matriz resistente para copias en plástico. Sus limitaciones están en la poca elevación, en la limitación de texturas $\mathrm{y}$ en la fragilidad del aluminio que puede romperse con facilidad.

Para la reproducción de representaciones táctiles en gran cantidad se pueden utilizar otros materiales y técnicas, entre ellos se destaca la serigrafía, técnica comúnmente aplicada para la producción de prendas de vestir.

Producir mapas utilizando la serigrafía (Figuras 5 y 6) permite la reproducción en gran cantidad lo que disminuye considerablemente el valor unitario del mapa. Las 

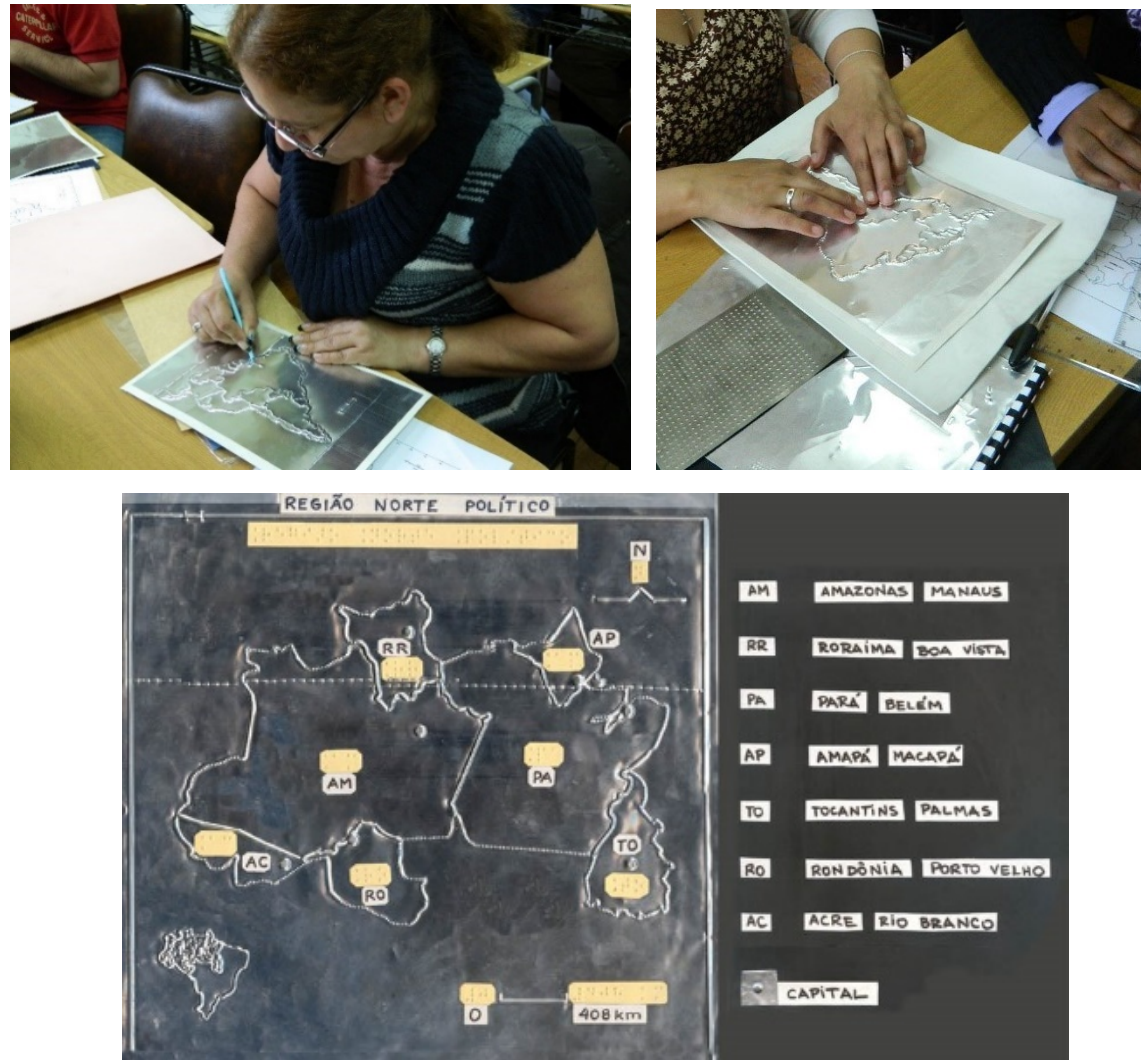

Figura 4a, b y c. Material táctil en láminas de aluminio, América del Sur, Región Norte de Brasil-Político.

Fuente: Acervo de Mapas Táctiles del LEMADI, USP y CECAT.

limitaciones se concentran en la resolución de la representación, las implementaciones lineales no pueden tener espesores finos pues no permiten el passo de la tinta por la pantalla; las elevaciones son limitadas (máximo $2 \mathrm{~mm}$ ) lo que disminuye el número de variables representables.

La tinta para tejido expansible con calor (puff) puede ser utilizada en pinturas artesanales, lo que aumenta las posibilidades de variación de las texturas y espesores de símbolos lineales y puntuales, pero vuelve a ser una técnica artesanal que aumenta el tiempo de producción de la representación y por lo tanto su costo.

\section{Mapas audiovisuales táctiles y tecnologías asistidas}

Con el desarrollo de la tecnología, otros materiales surgieron en el intento de facilitar la representación de mapas, gráficos e ilustraciones en relieve. Entre ellos se destaca 

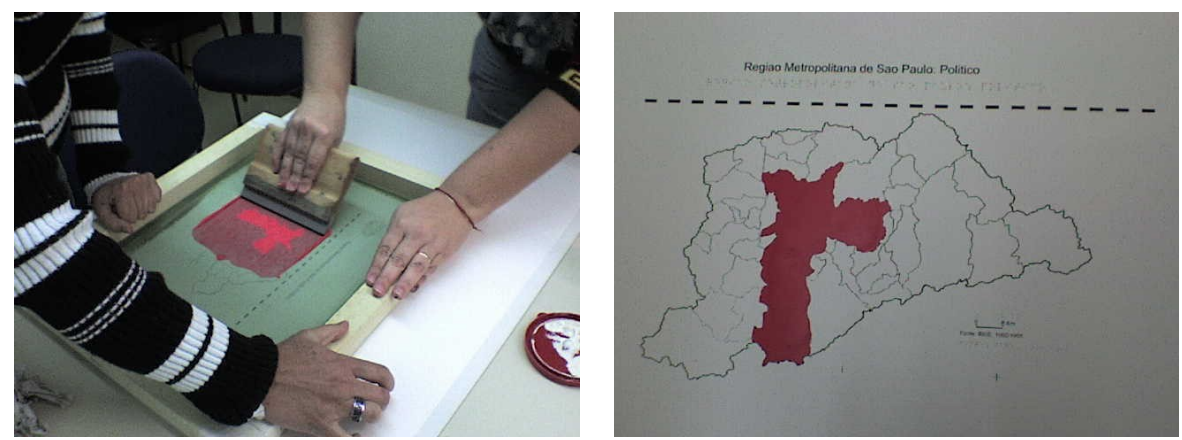

Figuras 5 y 6. Producción de mapa en serigrafía.

Fuente: Almeida, Carmo e Sena (2011: 368).

el papel micro capsulado (flexipaper), un papel especial que contiene dos capas de fibras, es impermeable y resistente, producido en varios países como Japón, Reino Unido, Australia y Estados Unidos. En este papel, la ilustración o el mapa se pueden imprimir en cualquier copiadora o impresora de inyección de tinta. Después del secado de la tinta, el papel pasa por algún equipo que produzca calor, lo que resulta en la elevación de las áreas en negro.

Esta técnica es muy utilizada para la impresión de informaciones virtuales, el usuario busca las imágenes en internet, imprime en el papel micro capsulado (Figura 7) y lo calienta, no necesitando así la impresora Braille para su reproducción.

La ventaja de este material es la facilidad de edición de los dibujos, ya que cualquier imagen se puede trabajar en el ordenador antes de la reproducción. Además, es un material resistente y ligero, lo que facilita su transporte y uso.

Debido a la limitación de la elevación de la impresión no es posible hacer una variación de texturas y alturas muy grande, lo que fuerza a una gran generalización de la información, pues sólo las impresiones en negro son elevadas con el calor.

La tecnología también ha permitido la producción de mapas táctiles a partir de matrices de madera (Figura 8) elaboradas en ruteadoras programables por $\mathrm{CNC}$ (Control Numérico Computadorizado) que permiten la transferencia con precisión de los mapas y demás representaciones gráficas elaboradas en SIG y softwares de diseño.

El costo de estas matrices es mayor que las producidas artesanalmente (Figura 9), pero presentan gran resistencia, posibilitando la obtención de más copias en plástico (Figura 10a y b). En este caso también hay limitaciones con relación a la altura y variación de las texturas, lo que refuerza la importancia de tener claro los objetivos y público al cual va dirigido el producto cartográfico antes de su confección.

Muchas veces, la elaboración de los mapas táctiles depende de la elección de los materiales, pues ciertas temáticas necesitan un número elevado de variables. Es por 


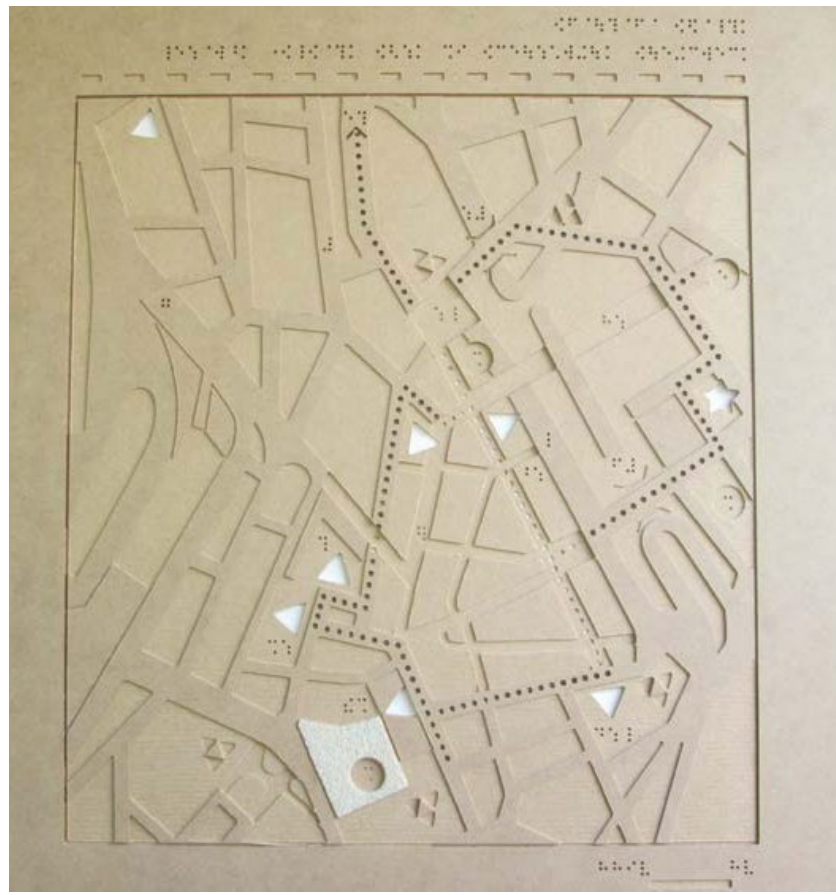

Figura 7. Máquina Fuser imprimiendo en papel microcapsulado y producción de mapa en papel micro capsulado (flexi-paper).

Fuente: CECAT-Almeida, Carmo e Sena (2011: 370).
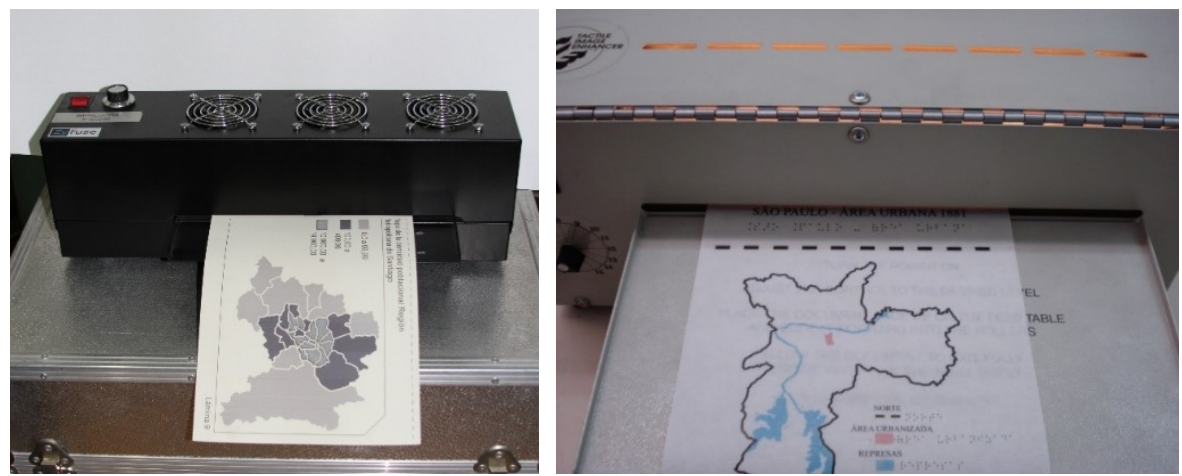

Figuras 8. Matriz en negativo producida en madera por medio de la máquina ruteadora. Circuito Turístico Sao Paulo.

Fuente: CECAT-UTEM (2016). 


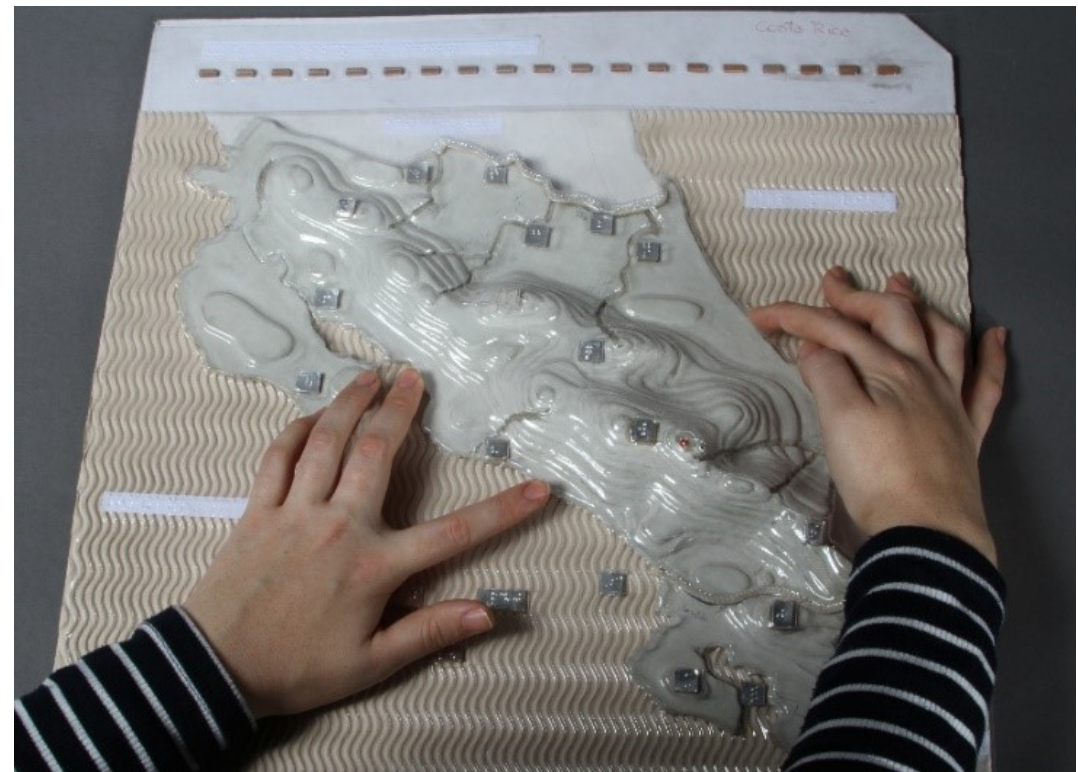

Figuras 9. Matriz producida artesanalmente correspondiente al mapa físico de Costa Rica y copia en plástico transparente, termoformado.

Fuente: CECAT-UTEM.
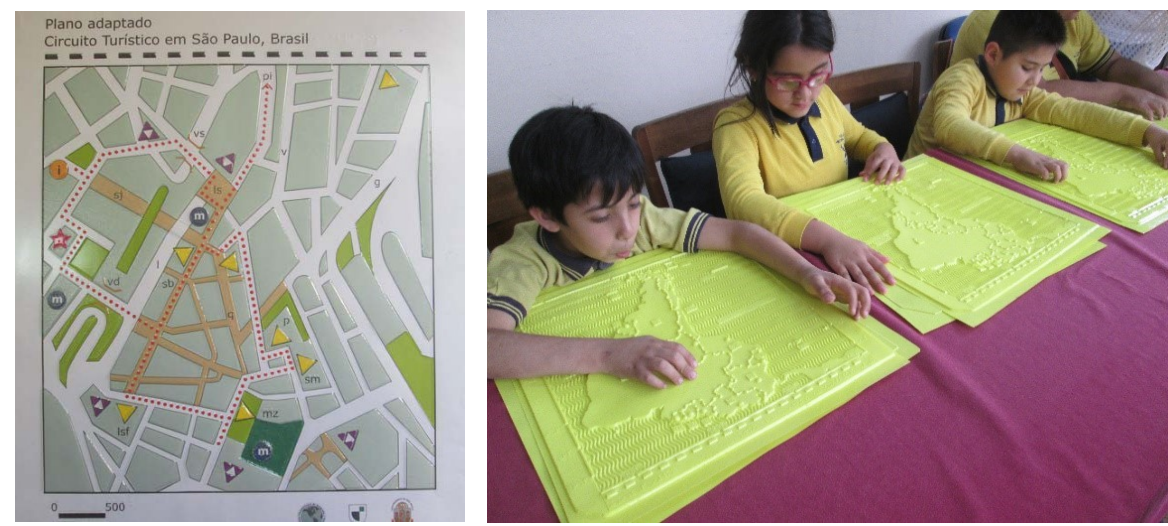

Figuras 10a y b. Termoformados, en transparente de Sector Turístico de Brasil y termoformado en plástico amarillo de mapa político de Sudamérica. Fuente: CECAT-UTEM (2016). 
esto que la persona que está realizando el producto cartográfico, necesita elegir si generaliza la información, agrupando datos y disminuyendo las variables en el mapa o se elabora una colección de mapas con más informaciones seleccionadas.

La tecnología ha sido aliada en el desarrollo de elementos de inclusión de la persona con discapacidad en la sociedad. El uso de sintetizadores de voz en las computadoras, el desarrollo de sitios adaptados y de sistemas en línea que evalúan la accesibilidad de las informaciones de la red, la elaboración de papeles para la impresión de representaciones en relieve son iniciativas que posibilitan mayor autonomía y ampliación del universo de ese grupo de ciudadanos. Además, maquetas construidas de forma artesanal pasaron a recibir sensores que permiten la inserción de sonidos y vibración, haciendo con que el material sea multisensorial.

Con la estructura de la maqueta construida (Figura 11) es posible añadir otra información sensorial, como sonidos programados por ordenador que se pueden accionar con el toque. Para esta inserción es necesario el uso de sintetizadores de voz y softwares que permitan la grabación y organización de las informaciones que serán insertadas.

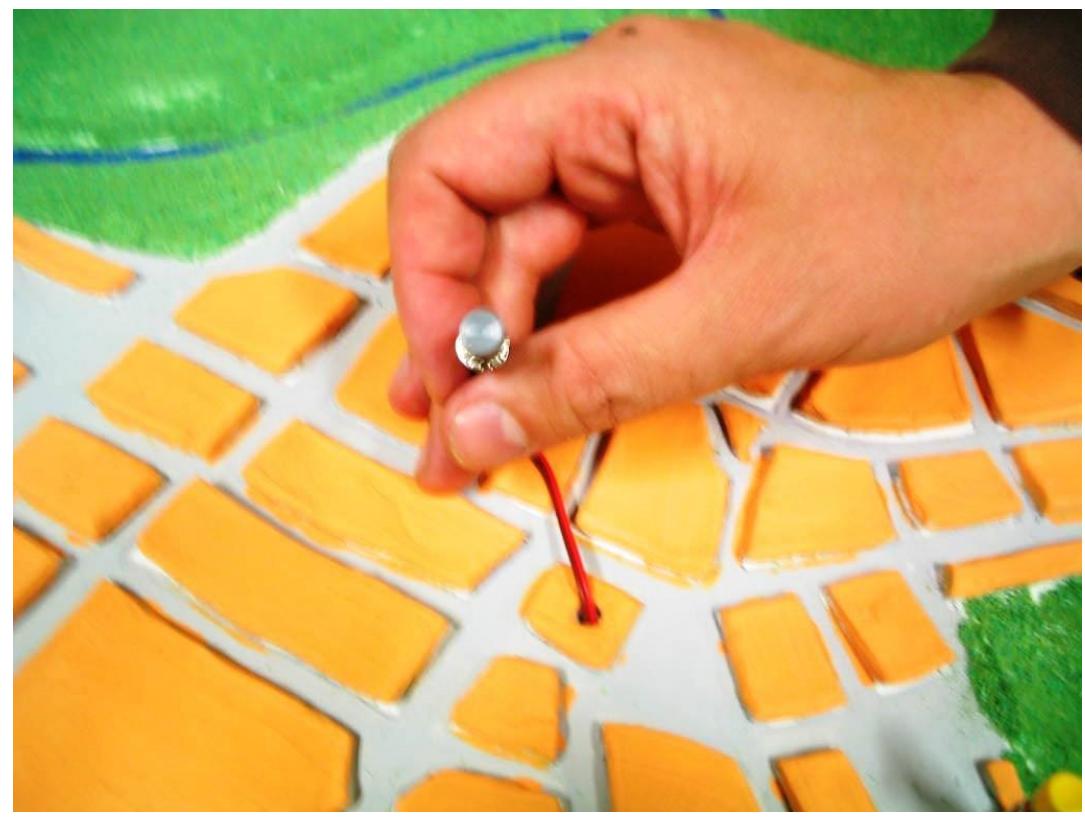

Figura 11. Sensor de tacto para inserción de sonidos en una maqueta táctil. Fuente: Sena (2008: 130). 


\section{Los símbolos táctiles}

Un mapa táctil se transforma es una herramienta fundamental en el apoyo de las personas con discapacidad visual, lo cual les permite acceder a información y conocimiento geográfico, por medio de la interpretación de los símbolos táctiles y escritura Braille. Un mapa táctil o producto cartográfico táctil tiene como objetivo representar de modo simple una imagen visual que eventualmente debe ser entendida por un discapacitado visual, por lo cual el mapa táctil debe ser cuidadosamente diseñado, puesto que de ello va a depender la capacidad de la persona en situación de discapacidad visual, para generar un mapa mental del sector y reconocer las interrelaciones que se puedan interpretar. La calidad del mapa depende del diseño, de la generalización, del tamaño y espaciamiento de los símbolos táctiles entre otros factores (Chung, 2018).

Hay que tener en cuenta que un mapa es una representación abstracta de un área específica, que se expresa por medio de los símbolos puntuales, lineales y areales (Coll, Barrientos, Huentelemu, 2017) que están basados en convenciones admitidas mediante acuerdos, transformándose en un registro simplificado de lo que se está representando, es decir es un modelo cartográfico, donde la persona con discapacidad podrá obtener mayor o menor información dependiendo de los conocimientos previos que posea de lo que se está representando en forma táctil y del grado de adiestramiento táctil que le permita reconocer mejor los símbolos convencionales representados.

La construcción y aplicación de los símbolos táctiles, requiere del conocimiento preciso de la forma y dimensiones que tendrá el símbolo que representará una información, por lo cual, para conseguirlo, es necesario guiarse por normas, especificaciones y metodologías en la selección y construcción de estos, y así lograr una correcta interpretación al utilizar correctamente las normas de construcción y de ubicación de los símbolos.

A continuación, se muestran unos ejemplos para la construcción y aplicación de símbolos puntuales (Cuadro 1), lineales (Cuadro 2) y areales (Cuadro 3) junto a productos cartográficos donde fueron utilizados.

Como se señaló anteriormente, los símbolos expresan una información y utilizarlos en forma estandarizada permitirá internalizar y conocer un conjunto de símbolos que, al ser analizados mediante el tacto, facilitarán la comprensión en forma efectiva y rápida de la información geográfica contenida en el mapa.

\section{Conclusiones}

Diseñar, construir e implementar un conjunto de simbologías táctiles para luego ser aplicadas en una cartografía, hace referencia a una forma de representación estandarizada, la cual facilitará la adaptación de mapas para personas con 


\section{Cuadro 1 \\ Símbolos puntuales}

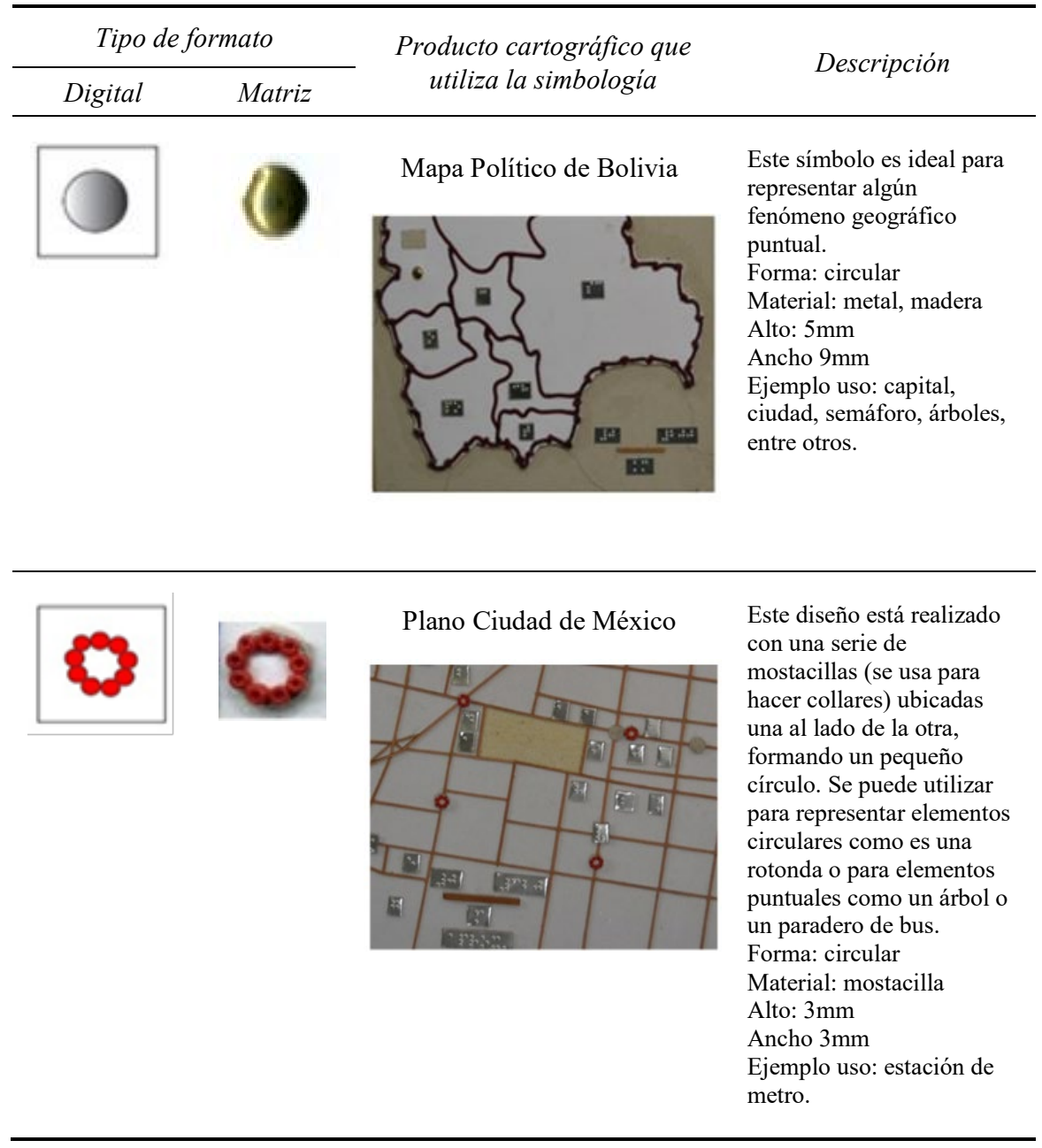

Fuente: CECAT. 
Cuadro 2

Símbolos lineales

\begin{tabular}{cll}
\hline Tipo de formato & $\begin{array}{c}\text { Producto cartográfico que utiliza } \\
\text { la simbología }\end{array}$ & \multicolumn{1}{c}{ Descripción } \\
\hline Matrizal & Plano Quito, Ecuador & $\begin{array}{l}\text { Una variable lineal, } \\
\text { como son las calles es } \\
\text { decir vías de } \\
\text { comunicación o límites, } \\
\text { se representan con } \\
\text { elemento que nos } \\
\text { permita mantener la } \\
\text { forma, como es un palo } \\
\text { de madera para } \\
\text { maquetas. Se debe } \\
\text { utilizar un material con } \\
\text { las características que se } \\
\text { señalan: } \\
\text { Forma: rectangular } \\
\text { Material: madera, metal } \\
\text { u otro } \\
\text { Alto: 1mm } \\
\text { Ancho 1mm } \\
\text { Ejemplo uso: calles de } \\
\text { una ciudad, línea férrea. }\end{array}$ \\
&
\end{tabular}

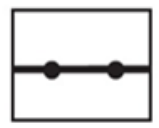

Mapa Político de Bolivia

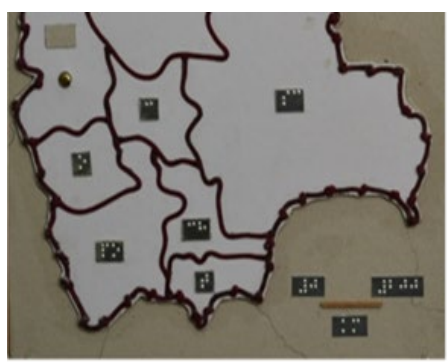

Para este tipo de diseño se utiliza un cordón en el cual se agregan nudos en forma equidistante, utilizándolo para alguna variable lineal como puede ser, un límite, una línea de tendido eléctrico. Se representan variables cualitativas que no dan información de cantidad ni jerarquía. Forma: lineal con puntos equidistantes Material: cordón, hilo grueso

Separación entre nudos: $1,5 \mathrm{~cm}$

Ejemplo uso: límite internacional

Fuente: CECAT. 


\section{Cuadro 3 \\ Símbolos areales}

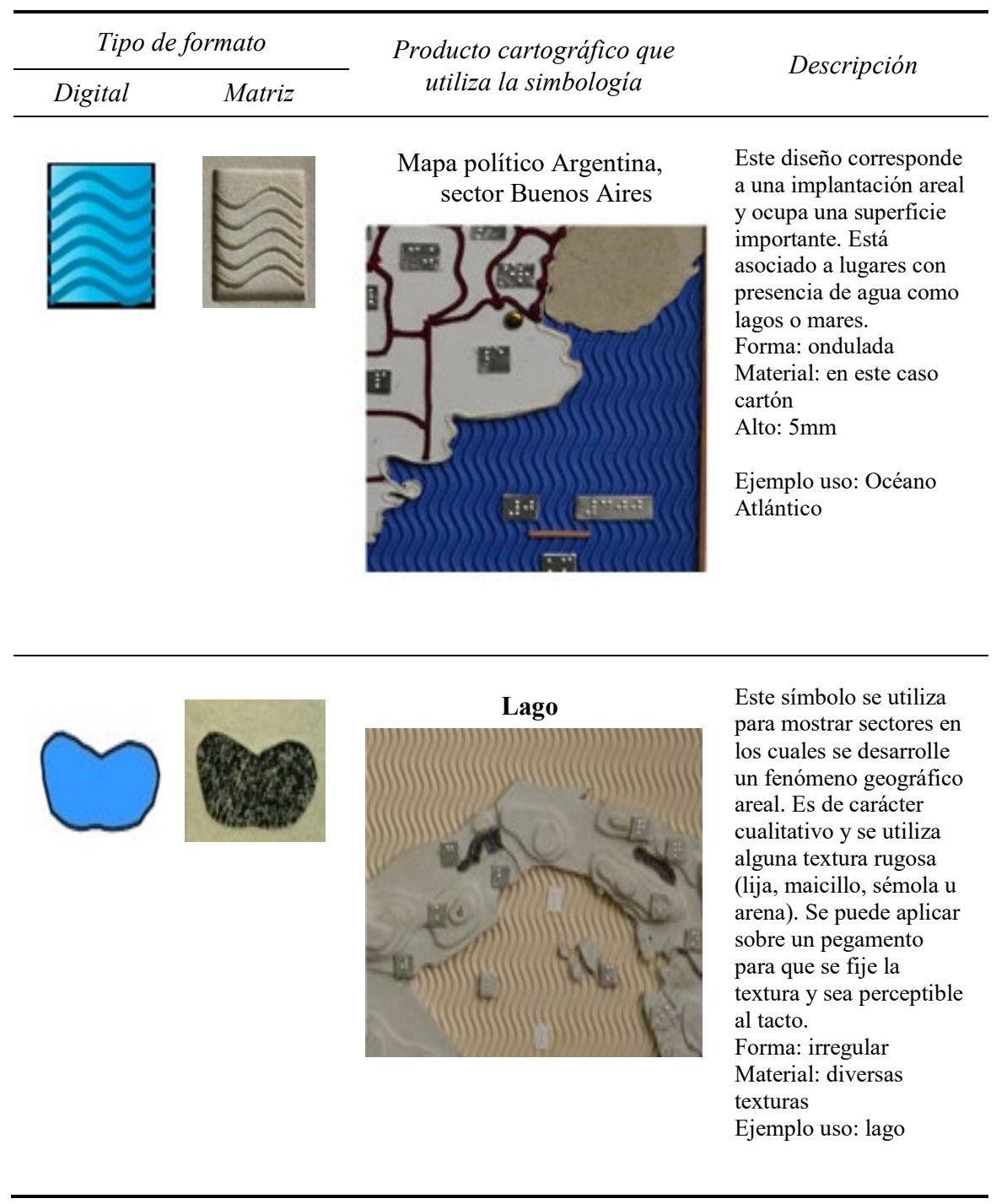

Fuente: CECAT. 
discapacidad visual, favoreciendo la inclusión al promover símbolos comunes para usuarios ciegos y con baja visión de los diferentes países, logrando un acceso al conocimiento de la cartografia.

En cuanto a la interpretación de un producto cartográfico (mapa, carta o plano) por parte de una persona ciega o de baja visión, podemos señalar que al utilizar simbología que cumpla con una serie de normas y estándares, facilitará el reconocimiento e interpretación de la información representada.

La implementación de diversas metodologías para la construcción de productos cartográficos utilizando diversas técnicas como las expuestas en este artículo, es una manera adecuada de entregar información a las personas en situación de discapacidad visual, con lo cual se brinda un servicio de calidad en el apoyo de la enseñanza. Las metodologías por si solas no van a mejorar la calidad, sin embargo, son una herramienta importante que le permite cumplir con las expectativas deseadas.

Un punto clave, es saber de las necesidades de las personas que utilizarán los productos cartográficos, con lo cual se logrará una eficiente comunicación de la información que está representada por medio de la simbología utilizada.

Finalmente, consideramos que una planificación de lo que se quiere lograr y cómo se va a hacer para conseguirlo, en cuanto al traspaso de información, se traducirá en los posibles caminos a seguir para alcanzar la implementación adecuada y construcción de los símbolos táctiles.

\section{Bibliografía}

Almeida, R.A. (2011). "Cartografia Tátil na USP: duas décadas de pesquisa e ensino", in Freitas, M.I.C.; Ventorini, S.E., Cartografia Tátil: orientação e mobilidade às pessoas com deficiência visual, Jundiaí - SP, Paco Editorial.

- (2014). "Inclusive Cartography: Theoretical and Applied Issues in Brazil", in Taylor, D.R.F., Lauriault, T.P. (eds.), Developments in the Theory and Practice of Cybercartography, Elsevier Science, chapter 8, pp. 107-128.

Almeida, R.A.; Carmo, W.R.E, Sena, C.C.R.G. (2011). "Técnicas Inclusivas de Ensino de Geografia" (capítulo 16), in Venturi, L.A.B., Geografia - Práticas de Campo, Laboratório e Sala de Aula, Editora Sarandi, São Paulo.

Basaraner, M. (2016). "Revisiting cartography: towards identifying and developing a modern and comprehensive framework", Geocarto International, vol. 31, no. 1, pp. 71-91. doi: http://dx.doi.org/10.1080/10106049.2015.1041560

Bertin, J. (1977). La Graphique et le Traitment Graphique de l'Information, França, Flamarion, $277 \mathrm{pp}$.

Centro de Cartografía Táctil (CECAT) (2016). Informe técnico final, Proyecto IPGH CART.02/GEOG.02 2016: "Propuesta de estandarización de simbología táctil 
para Latinoamérica: aplicación en cartografía turística", CECAT, Facultad de Humanidades y Tecnologías de la Comunicación Social, Universidad Tecnológica Metropolitana (UTEM), Santiago de Chile.

Chung, W. (2018). "Designing of Tactile map for blind people in Korea", International Journal of Civil Engineering and Technology (IJCIET), vol. 9, issue 11, pp. 2245-2251, Article ID: IJCIET_09_11_222. Recuperado de $<\mathrm{http}: / /$ www.iaeme.com/ijciet/issues.asp?JType=IJCIET \&VType=9\&IType=11 $>$.

Coll, A., Barrientos, T. y Huentelemu, V. (2017). Enseñando y aprendiendo el espacio geográfico por medio del tacto: orientaciones metodológicas, Ediciones Universidad Tecnológica Metropolitana, Santiago de Chile.

International Cartographic Association (ICA). Strategic Plan for the International Cartographic Association 2011-2019. Recuperado de <https://icaci.org/files/documents/reference_docs/ICA_Strategic_Plan_2011-2019.pdf $>$.

Sena, C. (2008). "Cartografia tátil no ensino de Geografia: uma proposta metodológica de desenvolvimento e associação de recursos didáticos adaptados a pessoas com deficiência visual”. Disertación de Doutorado, Facultad de Filosofia, Letras e Ciências Humanas, Universidad de São Paulo, São Paulo.

Vasconcellos, R. (1993). "A Cartografia Tátil e o Deficiente Visual: uma avaliação das etapas de produção e uso do mapa". Disertación de Doutorado, Facultad de Filosofia, Letras e Ciências Humanas, Universidad de São Paulo, São Paulo. (1996). "Tactile Mapping Design and the Visually Impaired User", in Wood, C. and Keller, P. (eds.), Cartographic Design: Theoretical and Practical Perspectives, John Wiley \& Sons, Inglaterra.

Virrantaus, K., Fairbairn, D., Kraak, M. (2009). "ICA Research Agenda", in The Cartographic Journal, vol. 46, no. 2, Maney Publishing, pp. 63-75 (13). 